\title{
Small Animal Models for Human Immunodeficiency Virus (HIV), Hepati- tis B, and Tuberculosis: Proceedings of an NIAID Workshop
}

\author{
Ramesh Akkina ${ }^{1}$, Daniel L. Barber ${ }^{2}$, Moses T. Bility ${ }^{3}$, Karl-Dimiter Bissig ${ }^{4}$, Benjamin J. Burwitz, \\ Katrin Eichelberg ${ }^{2}$, Janice J. Endsley ${ }^{6}$, J. Victor Garcia ${ }^{7}$, Richard Hafner ${ }^{2}$, Petros C. Karakousis ${ }^{8}$, \\ Brent E. Korba ${ }^{9}$, Rajen Koshy ${ }^{2}$, Chris Lambros ${ }^{2}$, Stephan Menne ${ }^{9}$, Eric L. Nuermberger ${ }^{8}$, \\ Alexander Ploss ${ }^{10}$, Brendan K. Podell ${ }^{1}$, Larisa Y. Poluektova ${ }^{11}$, Brigitte E. Sanders-Beer ${ }^{2, *}$, \\ Selvakumar Subbian ${ }^{12}$ and Angela Wahl ${ }^{7}$
}

${ }^{I}$ Department of Microbiology, Immunology and Pathology, Colorado State University, Fort Collins, Colorado, USA; ${ }^{2}$ National Institute of Allergy and Infectious Diseases (NIAID), National Institutes of Health, Bethesda, Maryland, USA; ${ }^{3}$ Department of Infectious Diseases and Microbiology, University of Pittsburgh, Pittsburgh, Pennsylvania, USA; ${ }^{4}$ Department of Pediatrics, Duke University, Durham, North Carolina, USA; ${ }^{5}$ Vaccine and Gene Therapy Institute, Oregon Health and Science University, Beaverton, Oregon, USA; ${ }^{6}$ Department of Microbiology and Immunology, University of Texas Medical Branch, Galveston, Texas, USA, ${ }^{7}$ Division of Infectious Diseases, Center for AIDS Research, University of North Carolina at Chapel Hill, North Carolina, USA; ${ }^{8}$ Division of Infectious Diseases, Center for Tuberculosis Research, Johns Hopkins University School of Medicine, Baltimore, Maryland, USA; ${ }^{9}$ Department of Microbiology \& Immunology, Georgetown University, Washington, DC, USA; ${ }^{10}$ Department of Molecular Biology, Princeton University, Princeton, New Jersey, USA; ${ }^{11}$ Department of Pharmacology and Experimental Neuroscience and Translational Mouse Model Core Facility, University of Nebraska Medical Center, Omaha, Nebraska, USA; ${ }^{2}$ The Public Health Research Institute Center of New Jersey Medical School, Rutgers University, Newark, New Jersey, USA

\begin{abstract}
ART ICLE H IS TORY
Received: October 20, 2019

Revised: November 27, 2019

Accepted: December 11, 2019

DOI:

$10.2174 / 1570162 X 18666191223114019$

Abstract: The main advantage of animal models of infectious diseases over in vitro studies is the gain in the understanding of the complex dynamics between the immune system and the pathogen. While small animal models have practical advantages over large animal models, it is crucial to be aware of their limitations. Although the small animal model at least needs to be susceptible to the pathogen under study to obtain meaningful data, key elements of pathogenesis should also be reflected when compared to humans. Well-designed small animal models for HIV, hepatitis viruses and tuberculosis require, additionally, a thorough understanding of the similarities and differences in the immune responses between humans and small animals and should incorporate that knowledge into the goals of the study. To discuss these considerations, the NIAID hosted a workshop on 'Small Animal Models for HIV, Hepatitis B, and Tuberculosis' on May 30, 2019. Highlights of the workshop are outlined below.
\end{abstract}

Keywords: HIV, AIDS, co-infections, HBV, tuberculosis, animal models.

\section{INTRODUCTION}

The meeting was convened to review the spectrum of small animal models for HIV and its frequently associated co-infections, hepatitis viruses and Mycobacterium tuberculosis (Mtb), which are in the portfolio of the Division of AIDS, NIAID. The purpose of the meeting was to gain insight into the types of research that can be carried out in small animals (Table 1) to generate meaningful data and to understand when it would be appropriate to switch to larger animals, such as nonhuman primates (NHP).

\footnotetext{
*Address correspondence to this author at the Division of AIDS, National Institute of Allergy and Infectious Diseases, National Institutes of Health, 5601 Fishers Lane, Bethesda, MD 20892-9830, USA; Tel: (240) 627-3209; E-mail: sandersbe@niaid.nih.gov
}

\section{SESSION 1: HIV AND EMERGING INFECTIONS}

J. V. Garcia introduced new mouse models that can support human respiratory viral infections in addition to HIV [1, 2]. An example is the human lung-only mouse (LoM) (Fig. 1) where the implanted human lung tissue transforms into a three-dimensional organoid with human lung structures, such as blood vessels, bronchial and bronchiolar airways, and cartilage. When injected directly into the organoid, Middle East Respiratory Syndrome Coronavirus (MERS-CoV) infects human epithelial, endothelial, and mesenchymal cells. Other applications of the LoM model shown were Zika virus (ZIKV), attenuated Mycobacteria (BCG), influenza A H3N2, respiratory syncytial virus, and human cytomegalovirus (HCMV). A. Wahl continued the presentation by introducing 
Table 1. Small Animal Models Presented for HIV, HBV, and Mycobacterium tuberculosis infection.

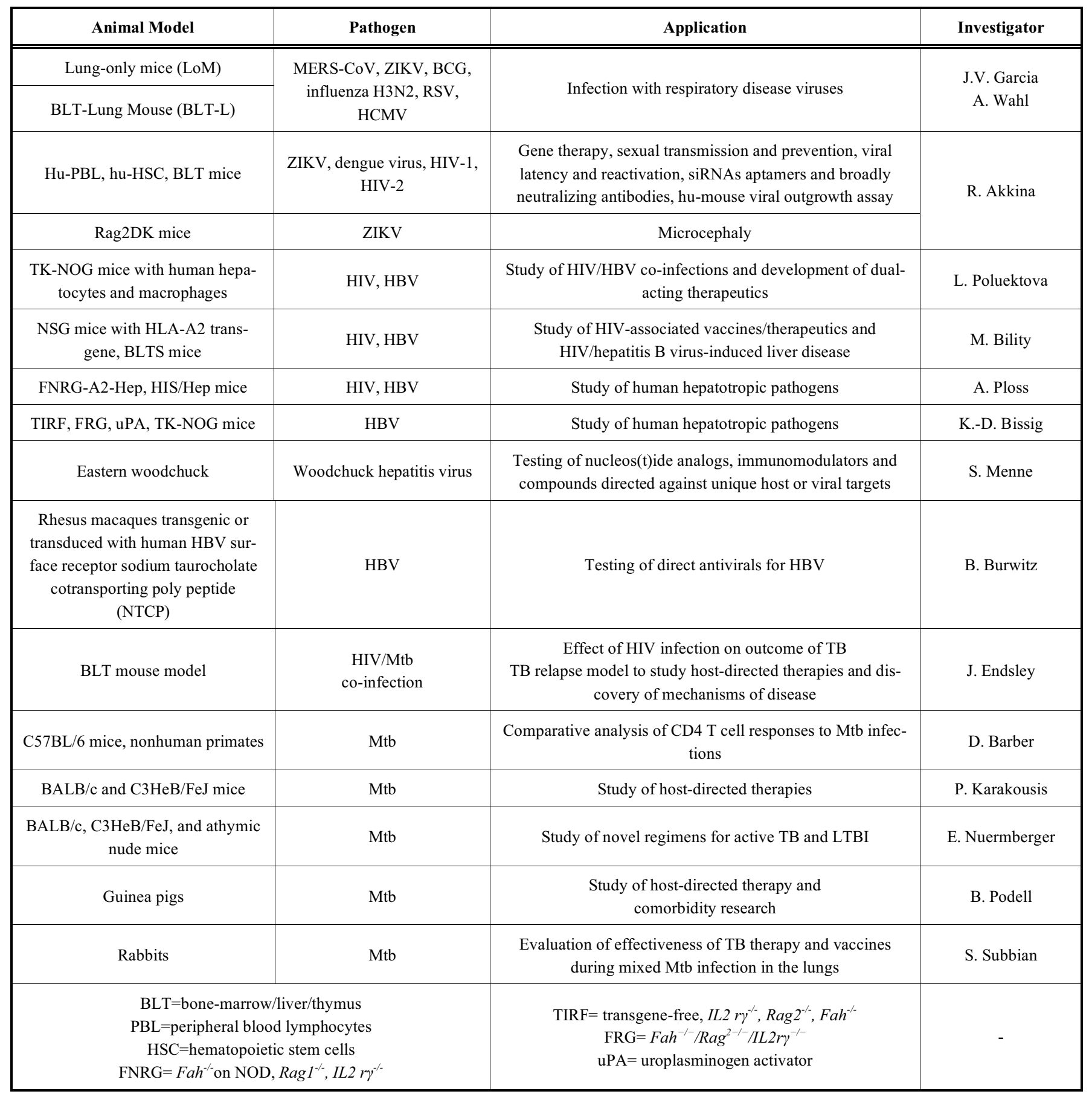

the BLT-Lung mouse. Similar to the BLT model [3-5], BLTLung mice became reconstituted with human CD45 cells in the peripheral blood and tissues. For comparison, LoM and BLT-Lung mice were injected with luciferase-encoding HCMV directly into the lung organoid, and the infection was monitored through bioluminescence imaging. BLT-Lung mice, due to the presence of cellular immune responses, were able to control CMV infection whereas LoM were not. CD8+ T cells expressed IFN $\gamma$ and CD107 $\alpha$, and CD4+ T cells expressed IFN $\gamma$ and TNF $\alpha$ following CMV peptide stimulation demonstrating that the $\mathrm{T}$ cells were functional. In addition,
HCMV neutralizing activity was detected in the plasma of the HCMV-exposed BLT-Lung mice. The BLT-Lung model could also potentially be used for HIV co-infections with TB or CMV.

R. Akkina's presentation highlighted the versatility of humanized mouse models for ZIKV, dengue virus, and HIV1 and HIV-2 infections. Since the presence of latent virus is a major obstacle for HIV cure, Dr. Akkina's laboratory developed an ultrasensitive in vivo viral outgrowth assay using humanized mice (hm-VOA assay) with higher sensitivity of detection than the commonly used in vitro quantitative VOA 


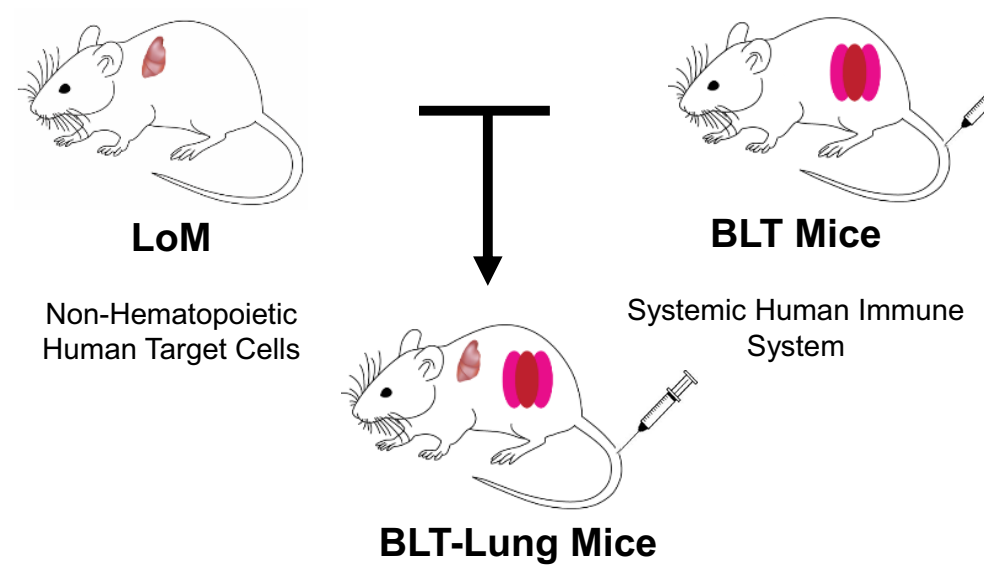

\section{Systemic Human Immune System \\ Non-Hematopoietic Human Target Cells \\ Autologous tissues}

Fig. (1). BLT/hu-Lung Mice. The BLT-Lung mouse model was created by combining the features of the Lung-only mouse (LoM) with the features of the BLT mouse. Thus, the BLT-Lung mouse model consists of both a systemic human immune system and non-hematopoietic human target cells.

$[6,7]$. Another example was the use of hu-mouse models for the testing of oral pre-exposure prophylaxis strategies (PrEP). Results demonstrated that synergistic as well as antagonistic pharmacokinetic (PK) aspects can be delineated when different combinations of drugs, such as maraviroc, raltegravir, and tenofovir, are administered [8]. While HIV-1 strains are extensively studied, pathogenesis and preclinical studies on HIV-2, which also induces AIDS, have been limited due to the lack of a suitable animal model. Dr. Akkina's work with HIV-2 infected humanized hematopoietic stem cell (hu-HSC) mice demonstrated that they experienced persistent viremia and CD4 T cell loss, i.e., hallmarks of AIDS. Results also revealed that combinatorial ART suppresses HIV-2 infection and latent virus re-emerges after treatment interruption [9]. Besides HIV, viral pathogenesis and immune responses to Zika virus (ZIKV) can also be evaluated in BLT mice [10]. Future work is directed towards developing a model for dengue secondary infection, which is an important risk factor for dengue hemorrhagic fever and shock syndrome.

\section{SESSION 2: HEPATITIS VIRUSES (HBV, HCV) AND HIV-HEPATITIS VIRUS CO-INFECTIONS}

L. Poluektova focused on HIV-HBV coinfection models for therapy. HIV and HBV display similarities in their replication cycles as they both have a reverse transcription (RT) step. Therapeutics approved for HBV mainly include nucleos(t)ide analogues inhibitors of RT, two of which (tenofovir and lamivudine) also have anti-HIV activity. She reported that Long-Acting Slow Effective Release antiretroviral therapeutics (LASER ART) control HBV replication in the liver of humanized mice $[11,12]$. In rats, a single injection of $75 \mathrm{mg} / \mathrm{kg}$ nanoformulated lamivudine persisted for 28 days in the peripheral blood with significant accumulation in spleen, liver, and lymph nodes. Similarly, a single injection of $75 \mathrm{mg} / \mathrm{kg}$ nanoformulated lamivudine suppressed HBV replication for 4 weeks in chronically HBV-infected TKNOG mice transplanted with human hepatocytes. To estab- lish an HIV/HBV co-infection model, TK-NOG mice were reconstituted with human hepatocytes as well as monocytederived macrophages [13]. It was found that the engrafted human cells suppressed HBV replication in hepatocyte transplanted mice; however, HIV infection counteracted the anti-HBV activity of the human cells. In summary, cotransplantation of hepatocytes and immune cells or combinations of antigen-presenting cells (APCs) and lymphocytes injected into mice can create a model for studying $\mathrm{HIV} / \mathrm{HBV}$ co-infections, supporting the development of dual-acting therapeutics.

M. Bility presented a humanized mouse model that is suitable for $\mathrm{HBV}$ infection by reconstituting immunodeficient A2/NSG mice [NOD.Cg-Prkdc(scid) Il2r $\gamma(\mathrm{tm} 1 \mathrm{Wjl}) / \mathrm{SzJ}$ mice with human HLA-A2 transgene] with human hematopoietic stem cells and liver progenitor cells (A2/NSG-hu HSC/Hep mice) $[14,15]$. He also discussed the bone marrow-liverthymus-spleen (BLTS)-humanized mouse model, which represents a significant improvement over existing models, with notably "improved" human myeloid cells, IgG secreting-B cells, and primary and secondary lymphoid tissues and human immunity $[16,17]$. Adding human skin and liver cell grafts to the BLTS mouse model facilitates the study of HIV vaccines and therapeutics and hepatitis $B$ virus-induced liver disease, respectively. Further enhancements of human xenograft animal models include immunodeficient rat and rabbit models, which may provide tools for developing robust small animal models for long-term studies of HIV comorbidities and associated end-organ disease.

Modeling (HIV-exacerbated) viral hepatitis in humanized mice was discussed by A. Ploss [18]. As mentioned earlier, human liver chimeric mice are a versatile model for studying human hepatotropic pathogens $[19,20]$. FNRG-A2 xenorecipients support robust co-engraftment with HLA-matched human hepatocytes and hematopoietic cells, and both FNRG-A2-Hep and human immune system (HIS)/Hep mice are susceptible to HBV infection (Fig. 2). FNRG-A2 HISHep dually engrafted mice mount an $\mathrm{HBV}$-specific CD8+ T 


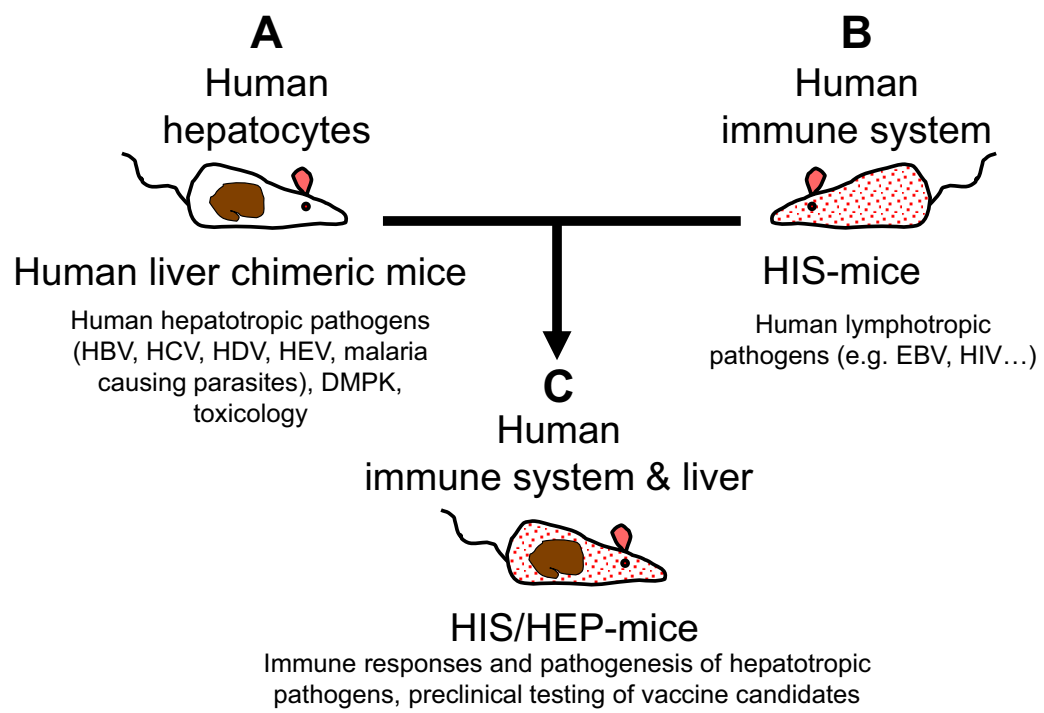

Fig. (2). Hu-Liver and HIS Mice. Dually engrafted mice have been generated by combining characteristics of human liver HEP-mice with features of human immune system mice. The HIS/HEP mice, therefore, allow susceptibility to human hepatotropic pathogens (HBV, HCV, HDV, HEV, etc.) and human lymphotropic pathogens (e.g., EBV, HIV) and can be used for evaluating the pathogenesis and immune responses of hepatotropic pathogens and for preclinical testing of vaccine candidates.

cell response, creating the possibility of immunophenotyping as hepatitis $\mathrm{B}$ core antigen ( $\mathrm{HBcAg}$ )-specific $\mathrm{CD} 8+\mathrm{T}$ cells acquire an activated phenotype. HBV viremia is consistently lower in these dually engrafted animals, suggesting some level of immune control by the co-engrafted HIS mice. Expectedly, at six weeks post-HBV infection, only minor liver damage was observed in HBV-infected FNRG-A2 HIS-Hep mice. However, further improvements are needed to mimic human immunity in these humanized mice.

K.-D. Bissig also presented studies of HBV replication in human liver chimeric mice [21, 22]. Three requirements need to be met for successful human hepatocyte transplantation: selection advantage of transplanted cells or selection disadvantage of endogenous cells, regeneration of the liver, and immunological tolerance. Human liver chimeric mice can be divided into genetic models (uPA, FRG, TK-NOG, PiZ, AFZ, etc.) or chemical models that utilize drugs to destroy mouse hepatocytes before transplantation of human liver cells. Limitations of the uPA mouse [23] include bleeding and neonatal death, a narrow window for transplantation of human hepatocytes, and a short life span after humanization. In the FRG [21] or transgene-free TIRF [24] mouse model (transgene-free, Il2 $\mathrm{rr}^{-/-}, \mathrm{Rag}^{-/-}, \mathrm{Fah}^{-/}$), which is based on a tyrosine metabolism defect, the drug NTBC (nitisinone) compensates for the FAH deficiency, and selection pressure for transplanted human hepatocytes can be applied by removing the compound [21]. These mice can achieve greater than $95 \%$ humanization of the liver [22] and can be used to study the pathogenesis of viral hepatitis and to evaluate the engraftment of stem cell-derived and diseased hepatocytes, hepatitis-related gene therapies, and the metabolism of hepatitis drugs [25]. Higher HBV titers correlate with higher human chimerism. Variability from mouse-to-mouse and the lack of a human immune system were mentioned as limitations of the human chimeric liver mice.
S. Menne introduced the Eastern woodchuck (Marmota monax) as an immunocompetent model for developing new HBV therapies [26, 27]. The woodchuck hepatitis virus (WHV), as well as HBV, belong to the genus Orthohepadnavirus in the family Hepadnaviridae. The genome structure, morphology and lifecycle of WHV and HBV are similar. Woodchuck infants infected with the strain WHV7 originating from Maryland became positive for WHV DNA and surface antigen (WHsAg), with peaks 8-12 weeks after inoculation. The majority of animals progress to chronicity $(60-75 \%)$ and develop hepatocellular carcinoma later in life. As in HBV infection, covalently closed circular DNA (cccDNA) is produced. Acute infection period during the first year is suitable for studying early pathogenic features. One to 2.5 years after infection is the ideal time frame for evaluating antiviral immunotherapies, and antitumor immunotherapies are usually tested 2.5 years post-infection. FDAapproved drugs, such as IFN $\alpha-2 b$, lamivudine, adefovir, entecavir, telbivudine, tenofovir, and other nucleos(t)ide analogs have been evaluated during their development phase in the woodchuck model. At present, the woodchuck model is used for testing immunomodulators (toll-like and RIG-I-like receptor agonists, AAV-encoded and recombinant IFN- $\alpha$ ), checkpoint inhibitors ( $\alpha$ PD-1), and compounds directed against unique host or viral targets [28, 29].

B. Burwitz is developing a rhesus macaque model of chronic HBV infection because chimpanzees are no longer available for this type of research. The HBV surface receptor, human sodium taurocholate cotransporting polypeptide (hNTCP), differs by five amino acids within the HBV binding region between humans and macaques. Due to one of these amino acid differences (G158R), HBV cannot bind to macaque NTCP. Dr. Burwitz demonstrated that macaque hepatocytes expressing hNTCP can support full HBV replication cycle following HBV binding and entry [30]. He concluded that his laboratory now has an HBV-susceptible primate model for testing direct-acting antivirals. 


\section{SESSION 3: MYCOBACTERIUM TUBERCULOSIS (MTB) INFECTION OR HIV-MTB CO-INFECTIONS}

J. Endsley has employed the BLT humanized mouse model for studying the complex molecular and immunological interactions of HIV/TB co-infection [31-33]. BLT mice develop poorly organized necrotic granulomas in response to Mtb infection. The subsequent induction of HIV co-receptors and proinflammatory response (IL- $1 \beta$ and $\mathrm{TNF} \alpha$ ) is associated with HIV replication at the TB granuloma site. HIV/TB co-infected mice have increased mycobacterial growth in the lung, and histologically the granulomas are larger and more inflamed compared to mice infected with Mtb alone. While exploring the mechanisms of these pro-inflammatory outcomes, Dr. Endsley found that mycobacterial exposure activates CLEC10a, a macrophage galactose-type lectin (MGL) selectively expressed on activated M2 macrophages and dendritic cells. However, CLEC10a is strongly downregulated in HIV/TB co-infected mice along with activation of proinflammatory response. Therefore, MGL may play an important immune role in TB through anti-inflammatory and/or antibacterial mechanisms because proinflammatory cytokines are induced in MGL knock-out mice infected with MTB [34] . Additionally, Dr. Endsley developed a small animal model of TB relapse as a result of HIV infection, in which BLT mice were infected with Mtb and, when they progressed to active disease, they were treated with rifampin and isoniazid for 2 months. Mice with paucibacillary Mtb infection following drug treatment were found to relapse upon infection with HIV-1 (JR-CSF).

D. Barber studied $\mathrm{T}$ cell migration/differentiation and spatial localization of CD4 T cells at sites of bacterial replication and identified new CD4 $\mathrm{T}$ cell molecules associated with protection against Mtb infection in mice and macaques. He pointed out that CD4 $\mathrm{T}$ cells are critical for the containment of TB because they provide help to Mtb-infected macrophages. In mice, less-differentiated CXCR3 + Th1 cells migrate into the lung parenchyma and protect against $\mathrm{Mtb}$ infection, while CX3CR1+ terminal effector cells accumulate in the blood vessels and do not contribute to control of the infection $[35,36]$. However unlike in mice, in monkeys, Mtb-specific CD4 $\mathrm{T}$ cells are largely CXCR3+ and CXCR3+CCR6+ $(\mathrm{Th} 1 *)$ cells, and neither develop a CX3CR1+ terminal effector phenotype nor accumulate in the blood vessels [37]. Collectively, it seems that mice make an overly Th1-polarized T-cell response during Mtb infection. Another aspect of Dr. Barber's research is the identification of new CD4 T-cell molecules that are associated with protection against Mtb infection. In mice, CD153 (CD30L) was preferentially expressed by protective lung parenchymal CD4 T cells and was required for host survival of Mtb infection as CD153-deficient mice succumbed early to Mtb infection [38]. Similar to mice, NHP and human Mtb-specific CD4 T cells expressed CD153 and its expression correlated with better outcomes. He concluded that there are major differences in the quality of T-cell polarization between mice and primates, leading to major differences in function and migration.

P. Karakousis showed interest in host-directed therapies (HDT) for TB since Mtb is known to subvert host responses and induce lung damage [39, 40]. Preclinical endpoints of
HDT include the assessment of bacterial clearance in the host and histological or radiographic assessment of lung inflammation. Although vastly underutilized, invasive spirometry and unrestrained whole-body plethysmography can be used to measure lung function in small animals. The conventional mouse model of TB chemotherapy has been employed to study the adjunctive activity of metformin [41], simvastatin [42, 43], and tofacitinib [44] (JAK inhibitor). Advantages include convenience, reduced cost, and availability of immunological reagents; however, mouse TB lesions (Fig. 3c) differ significantly from their human counterparts, raising the question of whether this model is predictive for studying the activity of novel HDT agents against TB. Specifically, mouse TB lesions are similar in cellular composition to human TB lesions, but tissue caseation and hypoxia and liquefaction/cavitation, pathological hallmarks of human $\mathrm{TB}$, are absent. To overcome these deficiencies, the $\mathrm{C} 3 \mathrm{HeB} / \mathrm{FeJ}$ mouse model, which lacks the $l p r 1$ gene, is increasingly being used as a preclinical drug screening model since these mice can develop necrotizing granulomas and even lung cavities upon Mtb infection [45, 46]. For example, ibuprofen adjunctive therapy resulted in significantly decreased tissue bacillary loads and increased survival in Mtbinfected $\mathrm{C} 3 \mathrm{HeB} / \mathrm{FeJ}$ mice. However, among all models presented, the NHP model is believed to reproduce most accurately the clinical, histological, and microbiological characteristics of human active and latent Mtb infection (LTBI) (Fig. 3a).

E. Nuermberger also relies on mouse models for $\mathrm{TB}$ regimen development with the goal of a) characterizing individual drug effects against $\mathrm{Mtb}, \mathrm{b}$ ) evaluating the activity of novel drug regimens (in models of active TB and LTBI), c) determining the contribution of individual drugs to multidrug regimens, and d) assessing the impact of caseous pathology (e.g., in $\mathrm{C} 3 \mathrm{HeB} / \mathrm{FeJ}$ mice) and immunodeficiency (e.g., in athymic nude mice). His laboratory developed a paucibacillary mouse model of LTBI to test the activity of clinical regimens, which accurately rank orders the regimens by recommended treatment duration in clinical use [47, 48]. He also found that the duration of treatment needed to cure $50 \%$ of mice gave a good estimate of effective treatment durations in clinical use. The model predicts the excellent activity of short-course bedaquiline treatment for LTBI, including for close contacts of multidrug-resistant TB patients. Third, he showed that BALB/c mouse models of active TB demonstrated utility and predictive accuracy in the development of novel regimens for active TB. Moreover, $\mathrm{C} 3 \mathrm{HeB} / \mathrm{FeJ}$ mice offer additional value for assessing the impact of caseation (Fig. 3d) on drug and regimen efficacy and may be a useful model for studying cavitation $[49,50]$. Progress has been made towards establishing a quantitative, PK/PD-based translational modeling and simulation platform for predicting clinical trial results using pre-clinical PK/PD and phase $1 \mathrm{PK}$ data [51].

According to B. Podell, a hallmark of the guinea pig model is the caseating granuloma lesion, which consists of a central area of necrosis surrounded by macrophages and a cuff of lymphocytes and fibroplasia (Fig. 3b). Granuloma lesions can be classified as 1) primary, classical granulomas that form before development of T-cell immunity and are dominated by TNF $\alpha$ and IL-12 cytokines, or 2) pneumonic-type secondary 


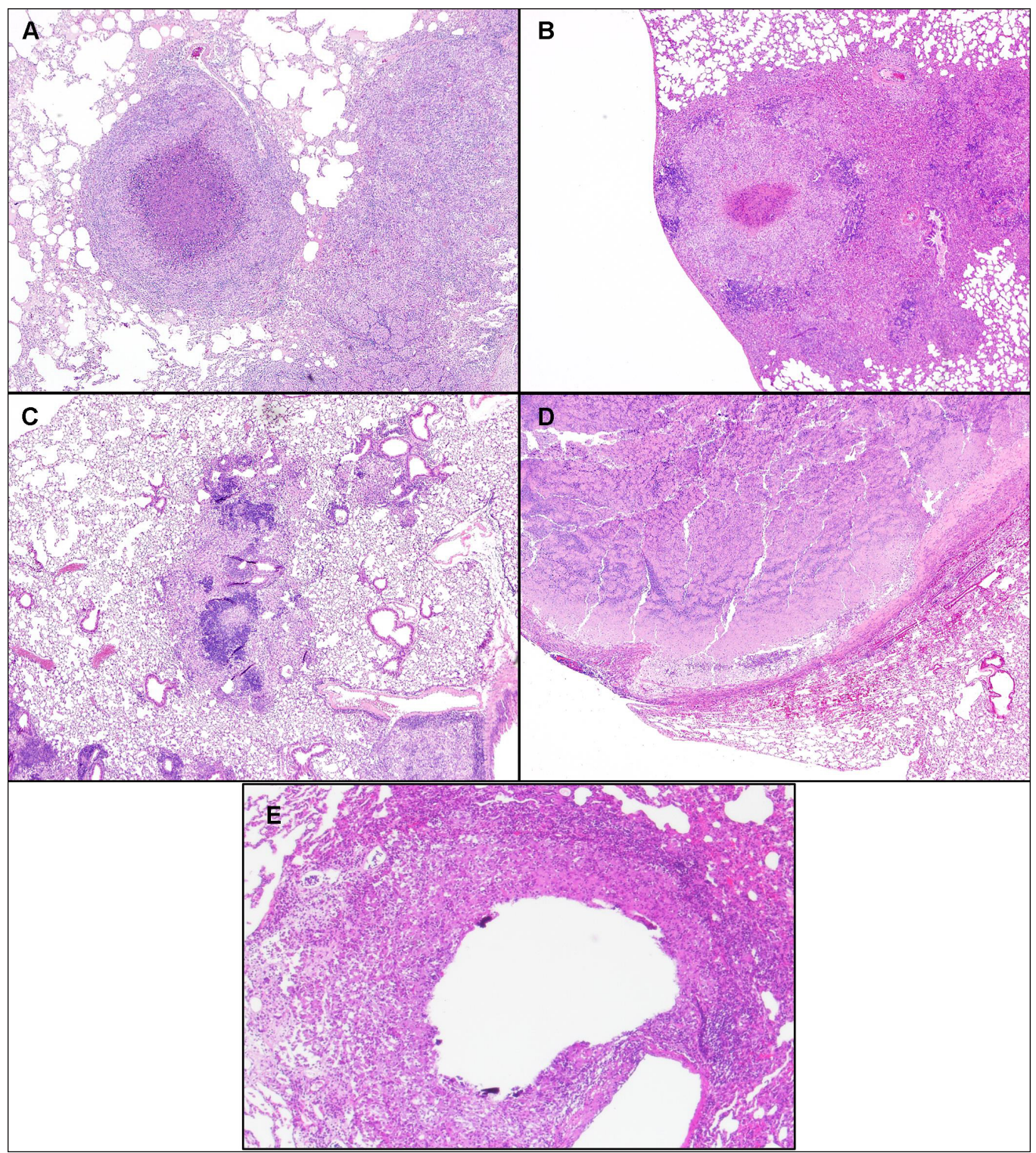

Fig. (3). Comparison of Mtb Lesions in Humans and Various Small Animal Models. The diversity of granulomas in various animal models of TB is shown. Lesions in rhesus macaques (A) appear histopathologically remarkably analogous to human granulomas consisting of a central area of necrosis with a wall of macrophages surrounded by a cuff of leukocytes. Similar structures also develop in guinea pigs (B). In stark contrast, C57BL/6 mice (C) do not develop highly organized TB lesions, and C3HeB/FeJ mice (D) develop highly suppurative lesions with cavitations more closely resembling abscesses than caseation. Similar to NHP, rabbits (E) develop lesions with cavitation resembling human pathology.

lesions that form after development of the cell-mediated response and are dominated by IFN $\gamma$ and regulatory cytokines, such as TGF $\beta[52,53]$. Due to necrotic lesions resembling human granulomas, this model has been primarily used for HDT, relapse models, and antimicrobial reduction [54]. In recent work, the guinea pig has also served as a model for comorbidity research to understand the mechanisms contributing to a higher risk of active TB disease and the impact of risk factors on TB disease outcomes. Moreover, guinea pigs are also frequently used for metabolic research because, unlike other rodents, they have glucose and lipid metabolism similar to humans [55]. Accordingly, guinea pig has contributed to the understanding of type 2 diabetes-TB comorbidity and represents an appropriate model for testing metformin or other repurposed metabolic drugs, which hold promise as adjunctive TB therapies [41]. Despite the value of this species in the development of new therapeutic strategies, the guinea pig has limitations in that this species does not reliably develop LTBI or cavitation [56].

S. Subbian presented a pulmonary Mtb infection model in rabbits, with clinical manifestations ranging from active disease with cavitation to paucibacillary infection (Fig. 3e and 4) $[57,58]$. Initially, two single clinical strains were used for challenge: the hypervirulent Mtb HN878, and the hyperimmunogenic Mtb CDC1551, which regresses into a latent stage. Rabbits infected with Mtb HN878 developed subpleural lesions at week 4 that further "matured" with cavities at week 16, mimicking the pathology of human pulmonary $\mathrm{TB}$, whereas Mtb CDC1551 infection seldom produced visible subpleural granulomas. However, the infected rabbits 


\section{Infectious Cycle of Mtb in Humans and Animal Models}

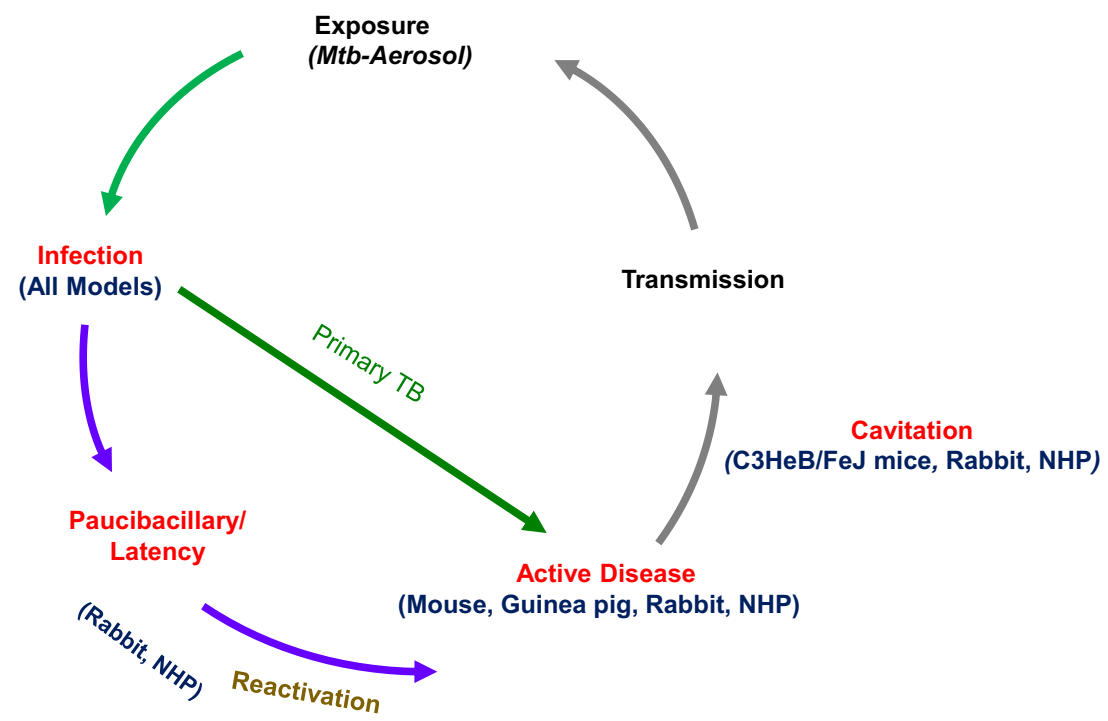

Fig. (4). Infectious Cycle of Mtb in Humans and Animal Models. This diagram shows the typical infectious cycle in mice, guinea pigs, rabbits, and nonhuman primates. All models develop primary TB and granulatomous responses ranging from granulomas resembling human pathology to pseudo-granulomas. Those from $\mathrm{C} 3 \mathrm{HeB} / \mathrm{FeJ}$ mice, rabbits, and NHP also evolve into cavitations that facilitate transmissions by aerosol. Paucibacillary infections and latency with the possibility of re-activation are also seen in rabbits and NHP.

developed LTBI spontaneously as defined by a lack of cultivable bacteria in the lung homogenates. This latent infection can be reactivated by immune suppression with triamcinolone $\left(\right.$ Kenalog $\left.^{\circledR}\right)$ or dexamethasone. Next, Dr. Subbian's group set up a pulmonary infection model in rabbits with mixed Mtb strains (Mtb HN878 and CDC1551) to understand the effect of dual infection on the host response. Dr. Subbian's team also examined the dose-dependent host response to MtbHN878 infection in rabbits with or without prior BCG-vaccination. His data demonstrated that the aerosol inoculum dose of Mtb determined the course of initial infection towards either progressive disease or manifestation of infection and establishment of LTBI. The protective efficacy of BCG vaccines was also directly impacted by the Mtb challenge dose. In summary, the rabbit model of active TB and LTBI displayed distinct pathological features relevant to respective human conditions.

\section{DISCUSSION}

During the 'HIV and Emerging Infections' session, clarification was requested whether the human lung in the BLTLung model is ventilated and whether the movement of the cilia and mucus secretions are present. It was explained that lung organoids can be maintained for over one year and all normal lung functions are preserved. GvHD development is donor-dependent and is mainly seen when male mice are transplanted with female human tissues. The CD4/8 ratios in the lung tissue are more human-like than mouse-like.

During the ' $\mathrm{HBV}$ ' session, questions pertained mostly to the generation of HBV humanized mouse models. Several transgenic and genetically modified immunodeficient mouse strains were presented that are invaluable for testing antiHBV therapeutics. For the HIV/HBV co-infection model, the simultaneous transplantation of human hepatocytes and human immune cells allows for modeling the interaction between the two viruses and the interaction of the viruses with the immune system. Moreover, liver sections from humanized mice carrying HBV and HIV are suitable for RNA transcriptome analysis, which can reveal the pathways of HBV replication control by immune cells. The drawback of a possible allogeneic reaction was resolved with the FNRG-A2 mouse model where HLA-type prescreened donors were used for engraftment; HLA-A2-positive hepatocytes and hematopoietic stem cells can also be combined for dual infection.

For 'Tuberculosis' session, interest was expressed in factors involved in Mtb re-activation in the HIV/TB coinfection model. Regarding the comparison of mouse and primate TB models, mice have IL-17 producing cells, but they are not bona fide Th17 cells. Also, the granulomas are histologically different between mice and primates. APC function in murine and primate systems is not well understood and was regarded as a potential gap area. Another issue raised was the timing of HDT and whether it should be administered before or after the TB-specific treatment. One observation that was made was that individuals with diabetes who were receiving metformin for at least three months before TB treatment was initiated showed increased survival rates. In summary, the questions raised were mostly of technical nature or regarded clarifications of scientific data presented at the meeting.

Concerning the limitations of various animal models [59, 60], humanized mouse models and primates are the only choices for HIV/SIV and HBV due to their narrow host range. Humanized mouse models are less expensive than primate models, and HIV/HBV rather than SIV or surrogate hepatitis viruses can be used for infection. However, their 
size, rate of metabolism, and longevity present some experimental challenges. More small animal model choices are available for TB, such as mouse, guinea pig, and rabbit models, some of which recapitulate human pathogenesis, but they do not recapitulate human (HLA-restricted) immune responses. However, TB infection in primates is still the model most comparable to humans.

\section{CONCLUSION}

Humanized mouse models are required for lympho- and hepatotropic infections, such as HIV and HBV and HIV/TB co-infections. Non-humanized animal models, such as C57BL6 and $\mathrm{C} 3 \mathrm{HeB} / \mathrm{FeJ}$ mice, guinea pigs, and rabbits can only accommodate Mtb infections, and not HIV or $\mathrm{HBV} / \mathrm{HCV}$.

\section{AUTHOR CONTRIBUTIONS}

R.A., D.L.B., M.T.B., K-D.B., B.J.B., J.J.E., J.V.G., P.C.K., S.M., E.L.N., A.P., B.K.P., L.Y.P., S.S., and A.W. presented original data at the workshop and edited the manuscript. B.E.S. organized the workshop and wrote the manuscript. K.E., R.H., B.E.K., R.K., and C.L. chaired sessions, contributed to the discussions, and edited the manuscript.

\section{CONSENT FOR PUBLICATION}

Not applicable.

\section{FUNDING}

The workshop was funded with federal funds from the National Institute of Allergies and Infectious Diseases, National Institutes of Health, Department of Health and Human Services, under contract no. HHSN272201100001G.

\section{CONFLICT OF INTEREST}

R.A., D.L.B., M.T.B., K-D.B., B.J.B., J.J.E., J.V.G., P.C.K., S.M., E.L.N., A.P., B.K.P., L.Y.P., S.S., and A.W. receive $\mathrm{NIH}$ funds for generating the presented data. B.E.S, K.E., R.H., B.E.K., R.K., and C.L. do not have any conflicts of interest.

\section{ACKNOWLEDGEMENTS}

The authors would like to thank Cynthia Soriano for her support of the workshop and Dr. Sandra Bridges for helpful discussions. In addition, the authors would like to thank NIAID Meet, in particular, Eric Delaney and Monica Washington, for help in organizing the workshop.

\section{REFERENCES}

[1] Wahl A, De C, Abad Fernandez M, et al. Precision mouse models with expanded tropism for human pathogens. Nat Biotechnol 2019; 37(10): 1163-73.

http://dx.doi.org/10.1038/s41587-019-0225-9 PMID: 31451733

[2] Honeycutt JB, Garcia JV. Humanized mice: models for evaluating NeuroHIV and cure strategies. J Neurovirol 2018; 24(2): 185-91. http://dx.doi.org/10.1007/s13365-017-0567-3 PMID: 28831774
[3] Kovarova M, Benhabbour SR, Massud I, et al. Ultra-long-acting removable drug delivery system for HIV treatment and prevention. Nat Commun 2018; 9(1): 4156.

http://dx.doi.org/10.1038/s41467-018-06490-w PMID: 30297889

[4] Denton PW, Garcia JV. Mucosal HIV-1 transmission and prevention strategies in BLT humanized mice. Trends Microbiol 2012; 20(6): 268-74.

http://dx.doi.org/10.1016/j.tim.2012.03.007 PMID: 22503637

[5] Wahl A, Swanson MD, Nochi T, et al. Human breast milk and antiretrovirals dramatically reduce oral HIV-1 transmission in BLT humanized mice. PLoS Pathog 2012; 8(6): e1002732.

http://dx.doi.org/10.1371/journal.ppat.1002732 PMID: 22737068

[6] Charlins P, Schmitt K, Remling-Mulder L, et al. A humanized mouse-based HIV-1 viral outgrowth assay with higher sensitivity than in vitro qVOA in detecting latently infected cells from individuals on ART with undetectable viral loads. Virology 2017; 507: 135-9.

http://dx.doi.org/10.1016/j.virol.2017.04.011 PMID: 28432928

[7] Schmitt K, Akkina R. Ultra-sensitive HIV-1 latency viral outgrowth assays using humanized mice. Front Immunol 2018; 9: 344. http://dx.doi.org/10.3389/fimmu.2018.00344 PMID: 29556230

[8] Thompson CG, Rosen EP, Prince HMA, et al. Heterogeneous antiretroviral drug distribution and HIV/SHIV detection in the gut of three species. Sci Transl Med 2019; 11(499): eaap8758. http://dx.doi.org/10.1126/scitranslmed.aap8758 PMID: 31270274

[9] Hu S, Neff CP, Kumar DM, et al. A humanized mouse model for HIV-2 infection and efficacy testing of a single-pill triple-drug combination anti-retroviral therapy. Virology 2017; 501: 115-8. http://dx.doi.org/10.1016/j.virol.2016.11.013 PMID: 27912079

[10] Schmitt K, Charlins P, Veselinovic M, et al. Zika viral infection and neutralizing human antibody response in a BLT humanized mouse model. Virology 2018; 515: 235-42.

http://dx.doi.org/10.1016/j.virol.2017.12.026 PMID: 29310105

[11] Gendelman HE, McMillan J, Bade AN, Edagwa B, Kevadiya BD. The promise of long-acting antiretroviral therapies: from need to manufacture. Trends Microbiol 2019; 27(7): 593-606. http://dx.doi.org/10.1016/j.tim.2019.02.009 PMID: 30981593

[12] Edagwa B, McMillan J, Sillman B, Gendelman HE. Long-acting slow effective release antiretroviral therapy. Expert Opin Drug Deliv 2017; 14(11): 1281-91.

http://dx.doi.org/10.1080/17425247.2017.1288212 PMID: 28128004

[13] Dagur RS, Wang W, Makarov E, Sun Y, Poluektova LY. Establishment of the dual humanized TK-NOG mouse model for HIVassociated liver pathogenesis. J Vis Exp 2019; (151).

[14] Bility MT, Cheng L, Zhang Z, et al. Hepatitis B virus infection and immunopathogenesis in a humanized mouse model: Induction of human-specific liver fibrosis and M2-like macrophages. PLoS Pathog 2014; 10(3): e1004032.

http://dx.doi.org/10.1371/journal.ppat.1004032 PMID: 24651854

[15] Cheng L, Li F, Bility MT, Murphy CM, Su L. Modeling hepatitis B virus infection, immunopathology and therapy in mice. Antiviral Res 2015; 121: 1-8.

http://dx.doi.org/10.1016/j.antiviral.2015.06.012 PMID: 26099683

[16] Samal J, Kelly S, Na-Shatal A, et al. Human immunodeficiency virus infection induces lymphoid fibrosis in the BM-liver-thymusspleen humanized mouse model. JCI Insight 2018; 3(18): 120430. http://dx.doi.org/10.1172/jci.insight.120430 PMID: 30232273

[17] Bility MT, Nio K, Li F, et al. Chronic hepatitis C infection-induced liver fibrogenesis is associated with M2 macrophage activation. Sci Rep 2016; 6: 39520.

http://dx.doi.org/10.1038/srep39520 PMID: 28000758

[18] Winer BY, Ding Q, Gaska JM, Ploss A. In vivo models of hepatitis B and C virus infection. FEBS Lett 2016; 590(13): 1987-99. http://dx.doi.org/10.1002/1873-3468.12157 PMID: 27009462

[19] Douam F, Ploss A. The use of humanized mice for studies of viral pathogenesis and immunity. Curr Opin Virol 2018; 29: 62-71. http://dx.doi.org/10.1016/j.coviro.2018.03.003 PMID: 29604551

[20] de Jong YP, Rice CM, Ploss A. New horizons for studying human hepatotropic infections. J Clin Invest 2010; 120(3): 650-3. http://dx.doi.org/10.1172/JCI42338 PMID: 20179350 
[21] Bissig KD, Le TT, Woods NB, Verma IM. Repopulation of adult and neonatal mice with human hepatocytes: A chimeric animal model. Proc Natl Acad Sci USA 2007; 104(51): 20507-11. http://dx.doi.org/10.1073/pnas.0710528105 PMID: 18077355

[22] Bissig KD, Wieland SF, Tran P, et al. Human liver chimeric mice provide a model for hepatitis $\mathrm{B}$ and $\mathrm{C}$ virus infection and treatment. J Clin Invest 2010; 120(3): 924-30.

http://dx.doi.org/10.1172/JCI40094 PMID: 20179355

[23] Dandri M, Burda MR, Török E, et al. Repopulation of mouse liver with human hepatocytes and in vivo infection with hepatitis B virus. Hepatology 2001; 33(4): 981-8.

http://dx.doi.org/10.1053/jhep.2001.23314 PMID: 11283864

[24] Barzi M, Pankowicz FP, Zorman B, et al. A novel humanized mouse lacking murine P450 oxidoreductase for studying human drug metabolism. Nat Commun 2017; 8(1): 39. http://dx.doi.org/10.1038/s41467-017-00049-x PMID: 28659616

[25] Bissig-Choisat B, Wang L, Legras X, et al. Development and rescue of human familial hypercholesterolaemia in a xenograft mouse model. Nat Commun 2015; 6: 7339.

http://dx.doi.org/10.1038/ncomms8339 PMID: 26081744

[26] Menne S, Cote PJ. The woodchuck as an animal model for pathogenesis and therapy of chronic hepatitis B virus infection. World $\mathrm{J}$ Gastroenterol 2007; 13(1): 104-24.

http://dx.doi.org/10.3748/wjg.v13.i1.104 PMID: 17206759

[27] Alioto TS, Cruz F, Gomez-Garrido J, et al. The genome sequence of the Eastern woodchuck (Marmota monax): A preclinical animal model for chronic hepatitis B. G3 (Bethesda) 2019; 9(12): 3943-52.

[28] Suslov A, Wieland S, Menne S. Modulators of innate immunity as novel therapeutics for treatment of chronic hepatitis B. Curr Opin Virol 2018; 30: 9-17. http://dx.doi.org/10.1016/j.coviro.2018.01.008 PMID: 29444493

[29] Korolowizc KE, Li B, Huang X, et al. Liver-targeted toll-like receptor 7 agonist combined with entecavir promotes a functional cure in the woodchuck model of hepatitis B virus. Hepatol Commun 2019; 3(10): 1296-310.

http://dx.doi.org/10.1002/hep4.1397 PMID: 31592075

[30] Burwitz BJ, Wettengel JM, Mück-Häusl MA, et al. Hepatocytic expression of human sodium-taurocholate cotransporting polypeptide enables hepatitis B virus infection of macaques. Nat Commun 2017; 8(1): 2146.

http://dx.doi.org/10.1038/s41467-017-01953-y PMID: 29247188

[31] Calderon VE, Valbuena G, Goez Y, et al. A humanized mouse model of tuberculosis. PLoS One 2013; 8(5): e63331.

http://dx.doi.org/10.1371/journal.pone.0063331 PMID: 23691024

[32] Nusbaum RJ, Calderon VE, Huante MB, et al. Pulmonary tuberculosis in humanized mice infected with HIV-1. Sci Rep 2016; 6: 21522.

http://dx.doi.org/10.1038/srep21522 PMID: 26908312

[33] Huante M, Nusbaum R, Endsley J. Co-infection with TB and HIV: converging epidemics, clinical challenges, and microbial synergy. 2019; pp. 123-53.

[34] Jondle CN, Sharma A, Simonson TJ, Larson B, Mishra BB, Sharma J. Macrophage galactose-type lectin-1 deficiency is associated with increased neutrophilia and hyperinflammation in gramnegative pneumonia. 2016; 196(7): 3088-96.

http://dx.doi.org/10.4049/jimmunol.1501790

[35] Sakai S, Kauffman KD, Schenkel JM, McBerry CC, Mayer-Barber $\mathrm{KD}$, Masopust $\mathrm{D}$, et al. Cutting edge: control of Mycobacterium tuberculosis infection by a subset of lung parenchyma-homing CD4 T cells. Journal of Immunology (Baltimore, Md : 1950) 2014; 192(7): 2965-9.

[36] Sakai S, Mayer-Barber KD, Barber DL. Defining features of protective CD4 T cell responses to Mycobacterium tuberculosis. Curr Opin Immunol 2014; 29: 137-42.

http://dx.doi.org/10.1016/j.coi.2014.06.003 PMID: 25000593

[37] Kauffman KD, Sallin MA, Sakai S, et al. Defective positioning in granulomas but not lung-homing limits CD4 T-cell interactions with Mycobacterium tuberculosis-infected macrophages in rhesus macaques. Mucosal Immunol 2018; 11(2): 462-73.

http://dx.doi.org/10.1038/mi.2017.60 PMID: 28745326

[38] Sallin MA, Kauffman KD, Riou C, et al. Host resistance to pulmonary Mycobacterium tuberculosis infection requires CD153 expression. Nat Microbiol 2018; 3(11): 1198-205. http://dx.doi.org/10.1038/s41564-018-0231-6 PMID: 30202016

[39] Kolloli A, Subbian S. Host-directed therapeutic strategies for tuberculosis. Front Med (Lausanne) 2017; 4: 171 . http://dx.doi.org/10.3389/fmed.2017.00171 PMID: 29094039

[40] Frank DJ, Horne DJ, Dutta NK, et al. Remembering the host in tuberculosis drug development. J Infect Dis 2019; 219(10): 151824 .

http://dx.doi.org/10.1093/infdis/jiy712 PMID: 30590592

[41] Singhal A, Jie L, Kumar P, et al. Metformin as adjunct antituberculosis therapy. Sci Transl Med 2014; 6(263): 263ra159.

http://dx.doi.org/10.1126/scitranslmed.3009885 PMID: 25411472

[42] Dutta NK, Bruiners N, Pinn ML, et al. Statin adjunctive therapy shortens the duration of TB treatment in mice. J Antimicrob Chemother 2016; 71(6): 1570-7.

http://dx.doi.org/10.1093/jac/dkw014 PMID: 26903278

[43] Dutta NK, Bruiners N, Zimmerman MD, et al. Adjunctive hostdirected therapy with statins improves tuberculosis-related outcomes in mice. J Infect Dis 2019; pii: jiz517.

http://dx.doi.org/10.1093/infdis/jiz517 PMID: 31605489

[44] Maiga M, Ahidjo BA, Maiga MC, et al. Efficacy of adjunctive tofacitinib therapy in mouse models of tuberculosis. EBioMedicine 2015; 2(8): 868-73.

http://dx.doi.org/10.1016/j.ebiom.2015.07.014 PMID: 26425693

[45] Vilaplana C, Marzo E, Tapia G, Diaz J, Garcia V, Cardona PJ. Ibuprofen therapy resulted in significantly decreased tissue bacillary loads and increased survival in a new murine experimental model of active tuberculosis. J Infect Dis 2013; 208(2): 199-202. http://dx.doi.org/10.1093/infdis/jit152 PMID: 23564636

[46] Apt A, Kramnik I. Man and mouse TB: contradictions and solutions. Tuberculosis (Edinb) 2009; 89(3): 195-8. http://dx.doi.org/10.1016/j.tube.2009.02.002 PMID: 19345146

[47] Nuermberger EL. Preclinical efficacy testing of new drug candidates. Microbiol Spectr 2017; 5(3).

http://dx.doi.org/10.1128/microbiolspec.TBTB2-0034-2017 PMID: 28643624

[48] Zhang T, Li SY, Williams KN, Andries K, Nuermberger EL. Shortcourse chemotherapy with TMC207 and rifapentine in a murine model of latent tuberculosis infection. Am J Respir Crit Care Med 2011; 184(6): 732-7.

http://dx.doi.org/10.1164/rccm.201103-0397OC PMID: 21659613

[49] Dartois V. The path of anti-tuberculosis drugs: From blood to lesions to mycobacterial cells. Nat Rev Microbiol 2014; 12(3): 15967.

http://dx.doi.org/10.1038/nrmicro3200 PMID: 24487820

[50] Lanoix JP, Ioerger T, Ormond A, et al. Selective inactivity of pyrazinamide against tuberculosis in $\mathrm{C} 3 \mathrm{HeB} / \mathrm{FeJ}$ mice is best explained by neutral $\mathrm{pH}$ of caseum. Antimicrob Agents Chemother 2015; 60(2): 735-43.

http://dx.doi.org/10.1128/AAC.01370-15 PMID: 26574016

[51] Bartelink IH, Zhang N, Keizer RJ, et al. New paradigm for translational modeling to predict long-term tuberculosis treatment response. Clin Transl Sci 2017; 10(5): 366-79. http://dx.doi.org/10.1111/cts.12472 PMID: 28561946

[52] Turner OC, Basaraba RJ, Orme IM. Immunopathogenesis of pulmonary granulomas in the guinea pig after infection with Mycobacterium tuberculosis. Infect Immun 2003; 71(2): 864-71. http://dx.doi.org/10.1128/IAI.71.2.864-871.2003 PMID: 12540568

[53] Ly LH, Russell MI, McMurray DN. Cytokine profiles in primary and secondary pulmonary granulomas of Guinea pigs with tuberculosis. Am J Respir Cell Mol Biol 2008; 38(4): 455-62. http://dx.doi.org/10.1165/rcmb.2007-0326OC PMID: 18032570

[54] Kiran D, Podell BK, Chambers M, Basaraba RJ. Host-directed therapy targeting the Mycobacterium tuberculosis granuloma: a review. Semin Immunopathol 2016; 38(2): 167-83.

http://dx.doi.org/10.1007/s00281-015-0537-x PMID: 26510950

[55] Fernandez ML. Guinea pigs as models for cholesterol and lipoprotein metabolism. J Nutr 2001; 131(1): 10-20.

http://dx.doi.org/10.1093/jn/131.1.10 PMID: 11208932

[56] Ernst JD. The immunological life cycle of tuberculosis. Nat Rev Immunol 2012; 12(8): 581-91.

http://dx.doi.org/10.1038/nri3259 PMID: 22790178 
[57] Subbian S, Tsenova L, Yang G, et al. Chronic pulmonary cavitary tuberculosis in rabbits: A failed host immune response. Open Biol 2011; 1(4): 110016.

http://dx.doi.org/10.1098/rsob.110016 PMID: 22645653

[58] Esteves PJ, Abrantes J, Baldauf HM, et al. The wide utility of rabbits as models of human diseases. Exp Mol Med 2018; 50(5): 66.

http://dx.doi.org/10.1038/s12276-018-0094-1 PMID: 29789565
[59] Allen TM, Brehm MA, Bridges S, et al. Humanized immune system mouse models: Progress, challenges and opportunities. Nat Immunol 2019; 20(7): 770-4.

http://dx.doi.org/10.1038/s41590-019-0416-z PMID: 31160798

[60] Akkina R, Allam A, Balazs AB, et al. Improvements and limitations of humanized mouse models for HIV research: NIH/NIAID "Meet the experts" 2015 workshop summary. AIDS Res Hum Retroviruses 2016; 32(2): 109-19.

http://dx.doi.org/10.1089/aid.2015.0258 PMID: 26670361 